\title{
Separating Heart Sound from Lung Sound Using LabVIEW
}

\author{
T E Ayoob Khan, Dr. P Vijayakumar
}

\begin{abstract}
Heart sounds interfere with lung sounds in a way that hampers the potential of respiratory sound analysis in terms of diagnosis of respiratory illness. This paper implements a VI for Heart Sound (HS) cancellation from Lung Sound (LS) records using the Advanced Signal Processing Toolkit of Lab VIEW 8.2. The method uses the multiresolution analysis of the wavelet approximation coefficients of the original signal to detect HS-included segments. Once the HS segments are identified, the method removes them from the wavelet coefficients and estimates the created gaps by using TSA ARMA modeling and prediction.
\end{abstract}

Index Terms-Virtual Instrument (VI), Multiresolution analysis (MRA), Time series analysis (TSA), Autoregressive Moving average (ARMA).

\section{INTRODUCTION}

Auscultation is one of the most important non-invasive and simple diagnostic tools for detecting disorders in the respiratory tract like lung diseases [1]. It is defined as the act of listening for sounds within the body, mainly for ascertaining the condition of the lungs, heart and other organs [2]. Diseases such as asthma, tuberculosis can be identified with this method through the analysis of lung and tracheal sounds.

Research on the diagnosis of respiratory pulmonary conditions like bronchitis, sleep apnea, asthma has established the utility of the stethoscope's acoustic signal in common day to day practice. However, despite their effectiveness, these instruments only provide a limited and subjective perception of the respiratory sounds. The drawbacks of using stethoscopes and listening to the sounds using the human ear are a) their inability to provide an objective study of the respiratory sounds detected, b) their lack of sufficient sensitivity and (c) the existence of the imperfect system of nomenclature [3].

In the last few decades, improvements in electronic recording and the development of computer-based methods have made quantitative studies of lung and tracheal sound signals possible as well as overcome many limitations of human ear subjective auscultation. Modern digital processing techniques, along with advancements in computer analysis, have become an established research method for the investigation of respiratory sounds.

Ayoob Khan T E is with the Electronics Engineering Department, College of Engineering, Kottarakkara, Kollam, Kerala, India (phone: +91-9447556722; e-mail: teakhan@yahoo.com).

Dr P Vijayakumar is the Professor and Head of Electrical Engineering Department, Karpagam College of Engineering, Coimbatore, India (phone: +91-9894994643; e-mail: vijay_p6@hotmail.com).
Computerized respiratory sound analysis can quantify changes in lung sounds, de-noise the signals of interest from any artifacts and nosiness, store records of the measurements made, and produce graphical representations of characteristic features of the respiratory sounds to help with the diagnosis and treatment of patients suffering from various lung diseases [4]. Since lung sounds have relatively low frequency and low intensity, it is essential to remove the noise and other interfering sounds (i.e., heart sounds) from the lung sounds prior to any diagnostic analysis.

Heart sounds interfere with lung sounds in a way that hampers the potential of respiratory sound analysis in terms of diagnosis of respiratory illness [5]. The features of lung sounds may be impure by heart sounds because lung and heart sounds overlap in terms of time domain and spectral content. High-pass filtering of lung-sound recordings to reduce heart sounds would remove important components of lung sounds.

This paper presents a method of lung sound analysis using the advanced signal processing tools of LabVIEW. The existing methods which are discussing here are not fully free from the artifacts of heart sounds. The software used in this method is having more flexiblility, it removes the heart sounds and predict the gaps successfully. It can also assist to general physicians to come up with more accurate and reliable diagnosis at early stages.

\section{HEART Sounds AND Lung Sounds}

Respiratory sounds present noninvasive measures of lung airway conditions [6]. However, features of lung sounds may be contaminated by heart sounds because lung and heart sounds overlap in terms of time domain and spectral content [7]. Heart sounds are clearly audible in lung sounds recorded on the anterior chest and may be heard to a lesser extent in lung sounds recorded over posterior lung lobes.

\section{A. Lung Sounds}

Lung sounds are produced by vertical and turbulent flow within lung airways during inspiration and expiration of air [5]. Lung sounds recorded on the chest wall represent not only generated sound in lung airways but also the effects of thoracic tissues and sound sensor characteristics on sound transmitted from the lungs to a data acquisition system [8]. Lung sounds exhibit a Power Spectral Density (PSD) that is broadband with power decreasing as frequency increases [5]. The logarithm of amplitude and the logarithm of frequency are approximately linearly related in healthy subjects provided that the signals do not contain adventitious sounds. As the flow in lung airways increases, sound intensity 
increases and several mathematical relations between lung sounds and airflow have been proposed [9]. It is important to note that inspiratory and expiratory lung sounds differ in terms of both amplitude and frequency range. At comparable flows, inspiratory lung sounds will exhibit greater intensity than expiratory sounds [9].

\section{B. Heart Sounds}

Heart sounds are produced by the flow of blood into and out of the heart and by the movement of structures involved in the control of this flow [10]. The first heart sound results when blood is pumped from the heart to the rest of the body, during the latter half of the cardiac cycle, and it is comprised of sounds resulting from the rise and release of pressure within the left ventricle along with the increase in ascending aortic pressure [10]. After blood leaves the ventricles, the simultaneous closing of the semi lunar valves, which connect the ventricles with the aorta and pulmonary arteries, causes the second heart sound.

The electrocardiogram (ECG) represents the depolarization and repolarisation of heart muscles during each cardiac cycle. Depolarization of ventricular muscles during ventricular contraction results in three signals known as the Q, R, and S-waves of the ECG [10]. The first heart sound immediately follows the QRS complex. In health, the last $30-40 \%$ of the interval between successive R-wave peaks contains a period that is void of first and second heart sounds [7].

Characteristics of heart sound signals have been assessed in terms of both intensity and frequency. Though peak frequencies of heart sounds have been shown to be much lower than those of lung sounds [11], comparisons between lung sound recordings acquired over the anterior right upper lobe containing and excluding heart sounds show that PSD in both cases is maximal below $150 \mathrm{~Hz}$.

\section{HS CANCELLATION}

During breathing lung sounds propagate through the lung tissue and can be heard over the chest wall. The tissue acts as a frequency filter with special characteristics based on pathological changes. Therefore, auscultation and acoustical analysis of lung sounds are primary diagnostic assessments for respiratory diseases. However, heartbeat produces an intrusive quasi-periodic interference sound that masks the clinical interpretation of lung sounds over the low frequency components. The main components of Heart Sounds (HS) are in the range $20-100 \mathrm{~Hz}$, in which the lung sound has major components. High Pass Filtering (HPF) with an arbitrary cut-off frequency between 70 and $100 \mathrm{~Hz}$ is not efficient in this case because lung sounds have major components in that region particularly at low flow rates. Therefore, HS reduction from lung sounds without altering the main characteristic features of the lung sound has been of interest for many researchers [12].

Accurate diagnosis of respiratory disease depends on understanding the different noises present in the lung sounds and remove them successfully. Heart sounds interfere with lung sounds in a manner that hampers the potential of respiratory sound analysis in terms of diagnosis of respiratory disease [5]. There are different methods that have been applied for filtering heart sounds from lung-sound recordings as those that require a separate noise reference and those that do not, namely linear adaptive filters and filters employing time-frequency based methods respectively. These methods are compared on the basis of reduction in heart sounds and alteration to lung sounds by filtering.

\section{A. Linear Adaptive Filters}

There are four main components to a linear adaptive filter: the input or "reference" signal; the output of the adaptive filter; the desired filter response or "primary" signal; and the estimation error, which is the difference between the filter output and desired response [13]. The term "linear" refers to the physical implementation of a linear adaptive filter, which employs the principle of superposition between its input and output signals. In reality, the internal structure of a linear adaptive filter is highly nonlinear. A recursive algorithm within the adaptive filter updates filter parameters with each iteration (in discrete time operation) so as to minimize the estimation error.

Noise cancellation and linear prediction are the two main classes of linear adaptive filters that have been applied to lung sound recordings for reducing heart sounds [13]. Linear prediction serves to develop a model of a signal based on its past or future values. In adaptive noise cancellation, the primary input contains both the noise to be removed by the adaptive filter, and the signal of interest. The reference signal represents the noise portion of the primary input; thus, the filter output is a signal that models the noise in the input, and the signal of interest is determined by subtracting the filter output from the primary input. Fig.1. shows the basic scheme of an adaptive noise cancellation filter.

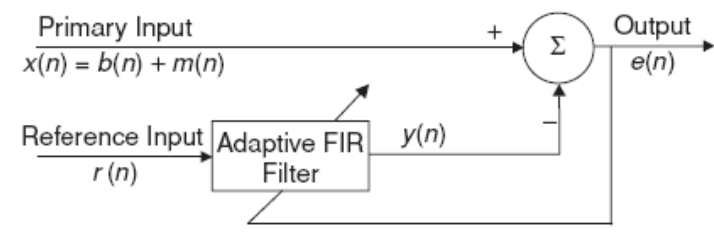

Fig.1. Block diagram of linear adaptive noise cancellation scheme. Symbols (as they pertain to heart sound cancellation from lung sounds): $b(n)=$ breath sounds; $m(n)=$ heart sounds; $r(n)=$ reference heart sounds; $y(n)=$ adaptive filter output; $\mathrm{e}(\mathrm{n})=$ denoised breath sounds.

The arrangement of the input samples and the updating formulas of adaptive filter parameters depend on the specific type of filter scheme used. All of the linear adaptive filters used for heart-sound reduction from lung sounds have employed adaptive filters with finite memory [13]. The most common form of a finite memory, or Finite-duration Impulse Response (FIR), filter is the transversal filter that consists of unit-delay elements that delay each of the $M$-samples of the input ( $M$ is the filter order); elements that multiply weights by input samples; and adders. Each sample, $k$, of the $M$-samples input reference vector, $r(n)$, that is multiplied by the conjugate of a weight value, $\omega_{k}$, and these products are summed to form the filter output $y(n)$ (Figure 1) [13]

$$
y(n)=\sum_{k=0}^{M} \omega_{k}^{*} r(n-k)
$$

The reduction in heart sounds via adaptive noise cancellation is dependent on the success of heart sound 
localization within the primary and reference inputs and the subsequent time alignment between these inputs and the choice of reference input. The stationarity of the data is another important consideration. Following schemes of adaptive filters are used: Least Mean Squares (LMS), Fourth-Order Statistics (FOS), Recursive Least Squares (RLS), Block Fast Transversal (BFT), and Reduced Order Kalman (ROK).

\section{B. Time Frequency Based Filtering}

Short-Time Fourier Transform (STFT) and wavelets provide means of examining signals simultaneously in both time and frequency domains [5]. STFT, or spectrogram, presents intensity of data within segments of constant time and frequency resolution. If data are nonstationary, window sizes must be chosen such that data within the windows are at least wide-sense stationary [5]. This limits window size and hence spectral resolution. Wavelet Transform (WT) analysis provides more flexibility in terms of resolution and does not require that data be stationary. Finding the WT of a signal $x(t)$ requires a mother wavelet function, $\psi(t)$, which is a waveform with morphological features that remain constant in terms of amplitude as it is dilated or compressed in time [5]. A wavelet family is defined using

$$
\psi_{s, \tau}(t)=\frac{1}{\sqrt{s}} \psi\left(\frac{t-\tau}{s}\right), s>0, \tau \in \mathfrak{R}
$$

Each member wavelet is described by a value of scale, $s$, which increases with the time span of the wavelet, and also by a translation parameter, $\tau$, which is the number of times the wavelet "traverses" a signal. A signal's WT is determined by multiplying the signal by the complex conjugate of a wavelet function, and integrating this product with respect to time [5]. Both STFT and WT have been used for noise cancellation schemes involving heart and lung sounds. One study considered the spectrogram of original lung sounds as an image and removed heart sounds using digital image processing [5].

To localize heart sounds, the spectrogram was thresholded within $10-110 \mathrm{~Hz}$ per breath phase using a threshold incorporating the mean and standard deviation of average power calculated for both inspiratory and expiratory lung sounds (separately). Segments that corresponded to heart sounds were either completely removed from the spectrogram, or band-stop filtered using a $20-300 \mathrm{~Hz}$ band; in each case, gaps were created. Two dimensional interpolation, accounting for frequency and phase, was applied within each gap to recreate lung sounds based on the lung sounds occurring before and after the gap. Time domain signals were reconstructed using inverse STFT, and results were assessed using PSD comparisons between filtered and unfiltered data.

A study involving heart-sound localization and removal from lung sounds using WT [14] also employed complete elimination of regions containing heart sounds as determined via thresholding. Three scales were used in wavelet decomposition with the fifth-order Symlet wavelet as the mother wavelet [14]. The product of two adjacent decomposition bands, or the "multiscale product," was calculated for heart sound localization, and five standard deviations plus the mean value of lung sounds outside of heart-sound regions defined the threshold for heart-sound localization. After removal of regions within each wavelet resolution level that corresponded to multiscale products that exceed the threshold, the lung sounds within these regions were modeled using either AR or MA modeling.

Wavelet-like multiresolution analysis for heart-sound localization was applied in another study in which subbands were created by filtering lung-sound recordings using half band low-pass and high-pass filters and decimating each filtered sequence by a factor of two [5]. This procedure was repeated for each low-pass-filtered subband, creating $N$ levels, each successive level having half the bandwidth, twice the frequency resolution, and half the time resolution of its predecessor, which is what results from WT as well. Using the time delay estimation scheme based on BFTF (block fast transversal filtering) [5], time delays were determined within subband levels four and five, which consistently matched. This delay was then applied to each of eight subbands of the reference heart-sound signal, and eight corresponding subbands of the primary input (lung sounds containing heart sounds) were adaptively filtered for heart-sound removal. The filter output and error signals per subband were reconstructed using an inverse scheme.

Daubechies quadrature mirror filters of eight coefficients were used for WT calculation in two studies employing wavelets for the removal of heart from lung sounds [15]. In that method, a threshold was applied to wavelet coefficients within each of 11 scales, which was defined by the standard deviation of the WT multiplied by a constant adjusting multiplicative factor chosen experimentally based on data recordings from individual subjects. The coefficients were separated per scale as those larger than the threshold and those smaller than the threshold. Reconstruction of coherent and residual signals from these groups of coefficients was performed across scales, representing the heart sounds (noise) and lung sounds (denoised data), respectively. The procedure was repeated in an iterative manner on the residual signals until the difference between the expected values of two successive residuals was less than a small constant (chosen as 0.00001). This stopping criterion implies that these waveforms were correlated and any coherent signal was no longer significantly present [5]. After the last iteration, the coherent parts obtained at each iteration of the procedure were summed, forming the heart sounds removed from lung sounds. The residual at the final iteration was considered the denoised lung sounds.

\section{Comparison of HS cancellation Methods}

Considering both quantitative and qualitative results, the Recursive Least Squares- Adaptive Noise Cancellation (RLS-ANC) filtering was superior over Wavelet TransformAdaptive Noise Cancellation (WT-ANC) for the signals. Both methods served to significantly reduce HS; however, the RLS-ANC preserved LS whereas the WT-ANC filtering altered them in terms of power and quality. Close inspection of the time domain data showed that neither method resulted in a reversal of the phase of LS. There is at least a ten-fold increase in processing time for the RLS-ANC over the WT-ANC, but this is insignificant if the loss of LS information is not prevented. Both methods succeeded in 
terms of HS localization. RLS-ANC requires a separate procedure to localize HS and apply these locations to the reference, whereas HS localization and separation may be performed in a single algorithm with WT-ANC [16].

\section{PROPOSED METHOD}

With advances in computer and software technology, Lung Sound (LS) computer-aided analysis has become part of a routine clinical assessment due to its diagnostic potentials. In respiratory sound recordings, Heart Sound (HS) is an unavoidable noise that has considerable overlap with the main components of LS over the frequencies below $150 \mathrm{~Hz}$. This technique presents a novel method for HS cancellation from LS records. The method uses the multiscale product of the wavelet coefficients of the original signal to detect HS-included segments. Once the HS segments are identified, the method removes them from the wavelet coefficients at every level and estimates the created gaps by using a set of linear prediction filters. The results were promising for HS removal from LS records and showed no hampering of the main components of the LS.

\section{A. Wavelet Multiscale Products}

The wavelet transform of a signal $f(\mathrm{x})$ is defined as

$$
W_{s}(x)=f(x) \otimes \psi_{s}(x)
$$

where $\Psi$ is an orthogonal wavelet, $s$ is the scale of the wavelet function,and $\otimes$ denotes convolution.

In [19], a different approach was proposed to obtain Ws(x). Let us consider the wavelet packet to be composed by the wavelet $\Psi$, and the scaling function $\theta$. The Discrete Wavelet Transform (DWT) algorithm states that the approximate form $A$ and the details $D$ from a signal $\boldsymbol{f}$ are given by the following equations:

$$
\begin{gathered}
A_{i+1}(x)=\left\langle f_{i}, \theta_{i+1}(x)\right\rangle \\
D_{i+1}(x)=\left\langle f_{i}, \psi_{i+1}(x)\right\rangle
\end{gathered}
$$

where $\langle.,$.$\rangle denotes the inner product, \boldsymbol{f}_{\mathrm{i}}$ is the projection of at the $\boldsymbol{i}$ th level, and $\boldsymbol{f}_{\mathbf{0}}=\boldsymbol{f}$.

As mentioned in [17], the product of two adjacent decomposition bands presents very interesting properties. Signal and noise have totally different behaviors in the wavelet domain. In [18], this behavior was analyzed using the concept of Lipschitz regularity. As an example, the Lipschitz regularity of a step function is 0 . If the function is smoother than a step then its Lipschitz regularity is positive. On the other hand, the Lipschitz regularity of the delta function is -1 . White Gaussian noise generally has a Lipschitz regularity of-1/2. [18].

The multiscale products are defined as

$$
P_{j, k}(x)=\prod_{i=k}^{k+j} W_{i} f(x)
$$

Where $k$ is a positive number and is equal or greater than $0, j$ is the desired number of levels to be multiplied, and $P_{j, k}$ is the multiscale product of order $j$ starting from the $k$ th level.

\section{B. Data Acquisition}

Data used in this study was lung sound recordings, in which lung sounds were acquired using a piezoelectric contact accelerometer (Siemens EMT25C) at the 3rd intercostals space anteriorly on the right upper lung lobe. Three lung sounds recordings took place according to three target flows: 7.5 (low), 15 (medium), and $22.5 \mathrm{ml} / \mathrm{s} / \mathrm{kg}$ (high). Each recording consisted of target breathing for $50 \mathrm{~s}$ followed by a 10 -s breath hold. Respiratory flow at the mouth was also recorded by a mouthpiece tube attached to a pneumotachograph and pressure transducer. Lung sound and flow signals were digitized simultaneously at $10240 \mathrm{~Hz}$ and 12-bits per sample (National Instruments DAQ) with custom written software in LabVIEW, while the flow signal was later decimated to $320 \mathrm{~Hz}$ [18].

\section{Heart Sound Localization}

As the first step of HS cancellation, the LS segments including HS must be identified. The premise of the proposed method in this paper is the HS localization technique presented in [14]. This approach relies on the different behaviors presented by the HS and LS across the wavelet scales. It uses the multiscale product at level 3 as a measure to detect singularities that in our case represent HS-included segments within the LS record. It is important to mention that the segment size that includes HS and is removed in this HS cancellation method is variable instead of being fixed sized blocks. This algorithm is explained in detail in [14].

\section{Linear Prediction Modeling}

Once the HS segments were localized and removed from the set of wavelet coefficients, the next step is to estimate the removed data. The length of the removed segment was different depending on the detected HS segment above the threshold. On average it was $100 \mathrm{~ms}$. In [18], estimating the removed data is done by linear prediction, using either Autoregressive (AR) or Moving Average (MA) models.

AR and MA models are two common signal processing tools used to predict past or future values of a time-limited signal. The predicted samples are basically weighted linear combinations of the signal known values. It is too simple to assume that lung sounds are stationary during the entire duration of a respiratory cycle (inspiration/expiration) especially at the vicinity of the onsets of breath. Therefore, correct selection of the order as well as the type of linear prediction model (AR or MA) must be done carefully to ensure the data used for prediction of the gaps are indeed stationary [18]. In this paper, regardless of the flow rate, ARMA modeling and prediction is used.

\section{Signal PROCESSING USING LABVIEW}

\section{A. Wavelet Analysis}

Advanced signal processing toolkit of LabVIEW 8.2 is used for processing the original LS including HS. During the first stage, the wavelet analysis tools are used for detection, localization and cancellation of HS included segments. Time series analysis tools are then used for modeling and predicting the HS removed portions of original LS. Wavelets are functions used to decompose signals. The wavelet transform computes the inner products of the analyzed signal 
and a family of wavelets.

Wavelets are localized in both the time and frequency domains, so wavelet signal processing is suitable for nonstationary signals, whose spectral content changes over time. The adaptive time-frequency resolution of wavelet signal processing enables to perform multiresolution analysis on nonstationary signals. The properties of wavelets and the flexibility to select wavelets make wavelet signal processing a beneficial tool for feature extraction applications.

The important signal processing applications of wavelets includes, analyzing signals at different scales, reducing noise, compressing data, and extracting features of signals. Multiscale analysis involves looking at a signal at different time and frequency scales. Wavelet transform-based multiscale analysis helps to understand both the long-term trends and the short-term variations of a signal simultaneously.

\section{B. Multiresolution Analysis}

Signals usually contain both low-frequency components and high-frequency components. Low-frequency components vary slowly with time and require fine frequency resolution but coarse time resolution. High frequency components vary quickly with time and require fine time resolution but coarse frequency resolution. Multiresolution Analysis (MRA) method is used to analyze a signal that contains both low and high frequency components. Wavelet signal processing is naturally an MRA method because of the dilation process.

The DWT is well-suited for multiresolution analysis. The DWT decomposes high-frequency components of a signal with fine time resolution but coarse frequency resolution and decomposes low-frequency components with fine frequency resolution but coarse time resolution. The central frequency and frequency bandwidth of the detail coefficients decrease by half when the decomposition level increases by one. For example, the central frequency and frequency bandwidth of $D 2$ are half that of $D 1$. The approximation at a certain resolution contains all of the information about the signal at any coarser resolutions. For example, the frequency band of $A 2$ covers the frequency bands of $A 3$ and $D 3$.

DWT-based multiresolution analysis helps to better understand a signal and is useful in feature extraction applications, such as peak detection and edge detection. Multiresolution analysis also removes unwanted components in the signal, such as noise and trend. The approximation at level 1 is the summation of the approximation and detail at level 2. The approximation at level 2 is the summation of the approximation and detail at level 3. As the level increases, lower frequency components will obtain, or large-scale approximation and detail, of the signal. Use the Multiresolution Analysis Express VI to decompose and reconstruct a signal at different levels and with different wavelet types [19].

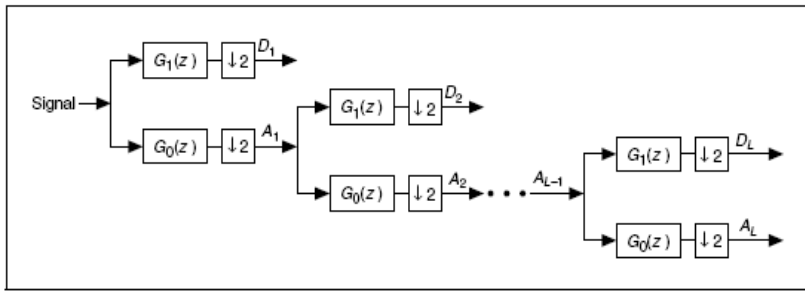

Fig.2. Discrete Wavelet Transform

\section{Time Series Analysis}

A time series is a sequence of observed values ordered through time. It is a sequence of observed values, for example, the air temperature in meteorological science, blood pressure in biomedical science, or vibration in mechanical engineering or civil engineering. Time series analysis uses a collection of systematic approaches to extract information about the characteristics of a physical system that generates time series. Approaches to time series analysis include estimating statistical parameters, building dynamic models, performing correlations, computing the power spectral density (PSD), and others. Single-source observations generate univariate time series, and multi-source observations form multivariate time series, or vector time series. If the statistical characteristic of a time series contains no systematic change, the time series is stationary. Otherwise the time series is nonstationary [19]. Time series analysis is useful to extract information from a time series, to discover the characteristics of a physical system that generates the time series, to predict the changes of a time series, or to improve control over the physical system.

Modeling and prediction methods are used to build dynamic models for a univariate or multivariate (vector) time series and perform predictions based on the estimated models. Building a model helps to understand the characteristics of a physical system that generates time series. Performing a prediction will helps to monitor and control the movements of a physical system. Using the Time Series Analysis Tools, we can build Polynomial models, Model parametric models and Stochastic models. Polynomial models includes autoregressive (AR) models, moving average (MA) models, and autoregressive-moving average (ARMA) models.

AR models of a time series enables to predict the current value $x_{t}$ of a time series, based on the past values $x_{t-1}, x_{t-2}, \ldots$, $x_{t-n}$, plus a prediction error. The parameter $n$ determines the number of past values used to predict the current value. The following (6) defines an AR model with an order of $n$ :

$$
x_{t}+a_{1} x_{t-1}+a_{2} x_{t-2}+\ldots+a_{n} x_{t-n}=e_{t}
$$

where $\left[1, a_{1}, a_{2}, \ldots a_{n}\right]$ are the AR coefficients and $e_{t}$ is the prediction error. Ideally, the residual prediction error is white noise with a mean value of zero.

Rewrite (6) more concisely as follows:

$$
A(q) x_{t}=e_{t}
$$

where $A(q)$ is the AR operator, which is defined as follows:

$$
A(q)=1+a_{1} q^{-1}+a_{2} q^{-2}+\ldots+a_{n} q^{-n}
$$

The term $q^{-k}$ is the backward shift operator, which is defined as follows:

$$
q^{-k} x_{t}=x_{t-k}
$$

The first step of estimating a model is to select an appropriate model. For a given model, selecting the model 
order is typically a trial-and-error process. Besides using background knowledge about the physical system that generates the time series, also needs to use other information, such as the information acquired from various statistical analysis methods, to justify the selected model order. One tool for determining the model order is the partial auto-correlation function of the time series. The partial auto-correlation function is a function of lag. The partial auto-correlation value becomes very small when the lag equals a suitable AR order.

Instead of computing the partial auto-correlation function for a time series, a set of model-selection criteria can be used to estimate the model order. From a least-square fitting standpoint, the higher the model order, the better the model fits the time series, because a high-order model has more degrees of freedom. However, an unnecessarily high-order may introduce spurious spectral artifacts in the resulting response. As a result, the criteria used to assess the model order therefore must not only rely on the model-fitting error but also incorporate a penalty when the order increases. Different selections for the penalty determine different criteria.

Akaike's Information Criterion: The Akaike's Information Criterion (AIC) is a weighted estimation error based on the unexplained variation of a given time series with a penalty term when exceeding the optimal number of parameters to represent the system. For the AIC, an optimal model is the one that minimizes the following equation:

$$
A I C=V_{n}\left(1+\frac{2 n}{L}\right)
$$

where $L$ is the number of data points in a time series, $n$ is the model order, and $V_{n}$ is the prediction error.

Bayesian Information Criterion: The Bayesian Information Criterion (BIC) replaces the term $2 n$ in the AIC with the expression $(n+n \ln (L))$. The BIC penalizes excess model order more severely than the AIC does. For the BIC, an optimal model is the one that minimizes the following equation:

$$
B I C=V_{n}\left(1+\frac{n+n \ln L}{L}\right)
$$

Final Prediction Error Criterion: The Final Prediction Error Criterion (FPE) estimates the model-fitting error when the model uses to predict new outputs. For the FPE, an optimal model is the one that minimizes the following equation:

$$
F P E=V_{n}\left(1+\frac{2 n}{L-n}\right)
$$

Minimal Description Length Criterion: The Minimal Description Length Criterion (MDL) is based on $\boldsymbol{V}_{\boldsymbol{n}}$ plus a penalty for the number of terms used. For the MDL, an optimal model is the one that minimizes the following equation:

$$
M D L=V_{n}\left(1+\frac{n \ln L}{L}\right)
$$

Phi Criterion: The Phi Criterion (PIC) generates an optimal model that minimizes the following equation:

$$
P H I=V_{n}\left(1+\frac{2 n \ln (\ln L)}{L}\right)
$$

Use the TSA AR Modeling Order VI to estimate a suitable AR model order for a time series. This VI implements the partial auto-correlation function and uses the AIC, BIC, FPE, MDL, and PIC methods to search for the optimal model order in the range of interest.

ARMA models are more accurate than AR models in approximating the response of linear systems with zeroes in the response transfer function. The following equation defines an ARMA model with an AR order of $n$ and the MA order of $m$ :

$x_{t}+a_{1} x_{t-1}+a_{2} x_{t-2}+\ldots+a_{n} x_{t-n}=e_{t}+b_{1} e_{t-1}+\ldots+b_{m} e_{t-m}$

Rewrite (15) more concisely as follows:

$$
A(q) x_{t}=B(q) e_{t}
$$

where $A(q)$ is the AR operator and $B(q)$ is the MA operator defined as follows:

$$
B(q)=1+b_{1} q^{-1}+b_{2} q^{-2}+\ldots . .+b_{m} q^{-m}
$$

Before estimating the ARMA model coefficients of a time series, specify a suitable order for the model is needed. Generally, specify an AR order of $n$ and an MA order of $n-1$, where $n$ is an integer. If the order of the response of the system that generates the time series is known, use that order directly. If the order is unknown, try orders from low to high to identify the lowest order in which the prediction error is sufficiently white. Use the TSA Whiteness Test VI to perform whiteness tests on the residual prediction error, by computing auto-correlation and the corresponding confidence limit for the auto-correlation. The variance of the prediction error also indicates if the ARMA model at a specified order fits the time series well. The smaller the variance of the prediction error is, the better the estimated model fits the time series. However, the variance of the prediction error for ARMA models decreases monotonically with order. Unnecessarily high orders introduce spurious artifacts in the resulting response if the measured time series is contaminated with noise or distortion. The changes in the variance of the prediction error that result from changes in the model order can indicate dynamic characteristics of a physical system that generates time series. This property is helpful in vibration monitoring applications. Use the TSA ARMA Prediction VI to predict the future values of univariate or multivariate time series based on the estimated ARMA or VARMA models [19].

\section{VI IMPLEMENTATION}

\section{A. System Block Diagram}

The VI is implemented using LabVIEW 8.2 version. Advanced signal processing toolkit is used for this purpose. Here Wavelet analysis tools and Time series analysis tools are used for separating HS from LS recordings. Fig. 3. shows the system block diagram. 


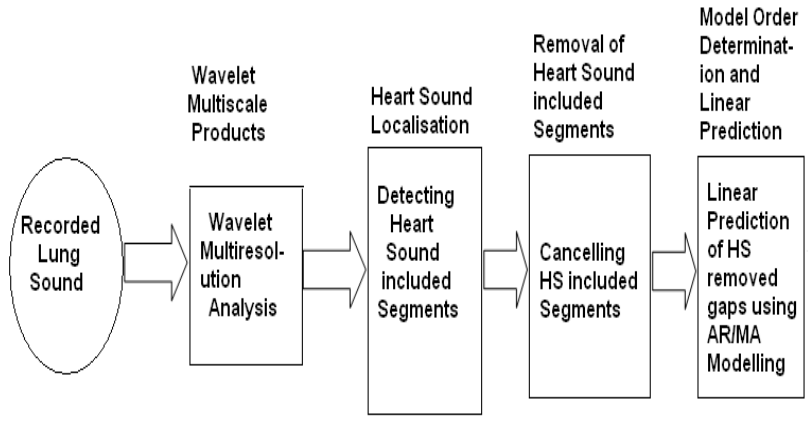

Fig. 3. System block diagram

The idea of this paper was to apply the Discrete Wavelet Transform (DWT) to the original LS record and locate HS segments automatically and accurately using multiscale products, removing those segments from each level of the wavelet coefficients, estimate the removed segments by ARMA modeling of previous data segments.

\section{B. Data Acquisition}

Data used in this study, lung sounds, were acquired with a piezoelectric contact accelerometer (Siemens EMT25C) at the 3rd intercostals space anteriorly on the right upper lung lobe. Lung sounds were digitized at $10240 \mathrm{~Hz}$ and 12-bits per sample (National Instruments DAQ) with a custom written software in LabVIEW.

Lund sound recordings are used as the input of this VI. These inputs are in .wav format which is read using Sound File Read Simple.VI.

\section{Heart Sound Detection}

The detection of heart sounds are done by the multiscale product of wavelet approximation coefficients. Multiresolution analysis.VI is used for this purpose, which is an express VI. Three scales were used in wavelet decomposition with the fifth-order Symlet wavelet as the mother wavelet.

The product of two adjacent decomposition bands presents very interesting properties. Signal and noise have totally different behavior in the wavelet domain. In [18], this behavior was analyzed using the concept of Lipschitz regularity. The multiplication of the DWT coefficients between the decomposition levels can lead to identification of singularities [18]. In the case of HS detection, the multiscale product of the wavelet coefficients of the original LS record is used to identify the HS segments within the LS signal. Multiresolution Analysis VI decomposes the signal according to the level we specify and reconstructs the signal from the frequency bands we select. Fig. 4 is the arrangement for finding multiscale product of wavelet approximation coefficients.

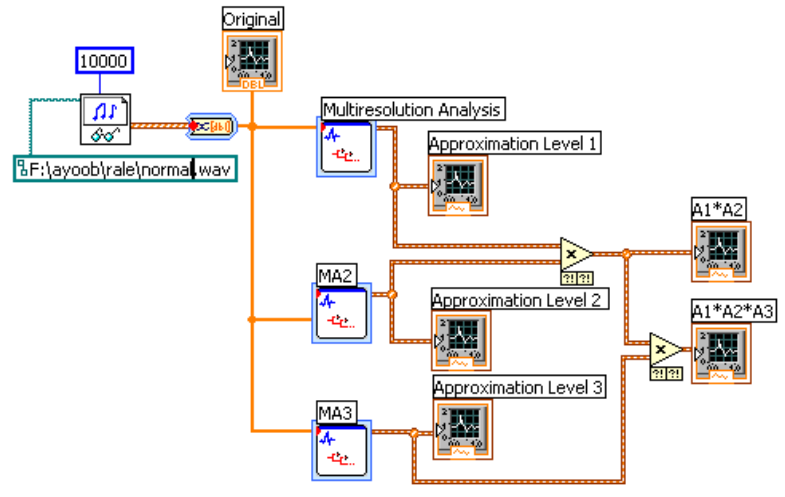

Fig. 4. Block diagram for heart sound detection.

\section{Heart Sound Cancellation}

The DWT of the original LS record was obtained using the Symlet (order 5) wavelet, which is a compactly supported wavelet with least asymmetry and decomposing the signal into 3 levels. Then, the product of the wavelet coefficients was calculated.

The cancellation of heart sounds is done by applying a threshold such that,

$$
\begin{gathered}
A_{i}^{\text {Th }}(n)=\left\{\begin{array}{cc}
A_{i}(n) P_{j}(n)<T h, \\
0 & \text { Otherwise }
\end{array}\right. \\
\text { where } \quad \mathrm{Th}=(\mu \pm 5 \sigma)
\end{gathered}
$$

where $A_{i}(\mathrm{n})$ and $A_{i}^{\mathrm{Th}}(\mathrm{n})$ are the original and thresholded wavelet approximation coefficients at level $k$, respectively; and $T h$ is the threshold value simply chosen to be above the mean plus or minus 5 times the standard deviation of the portions of original LS free of HS. To extract HS free portions of LS recording, we have to use an express VI named Extract Portion of Signal.

Fig. 5 is the block diagram for removing the HS included segments from the original LS including HS. The HS locations were detected accurately and removed from the DWT coefficients of the original LS record.

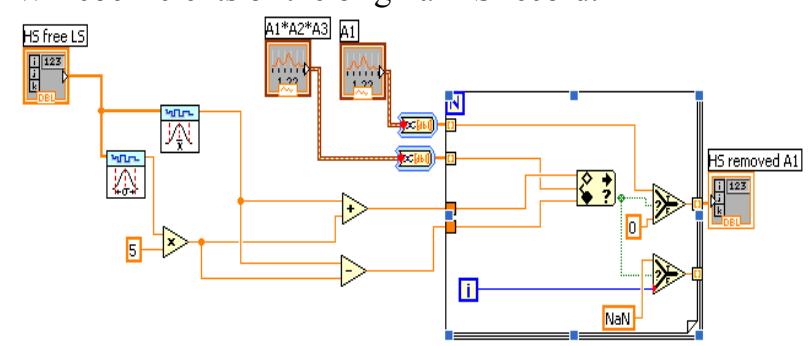

Fig. 5. Block diagram for removal of HS

Fig. 6. shows the block diagram for locating the heart sound removed portions.

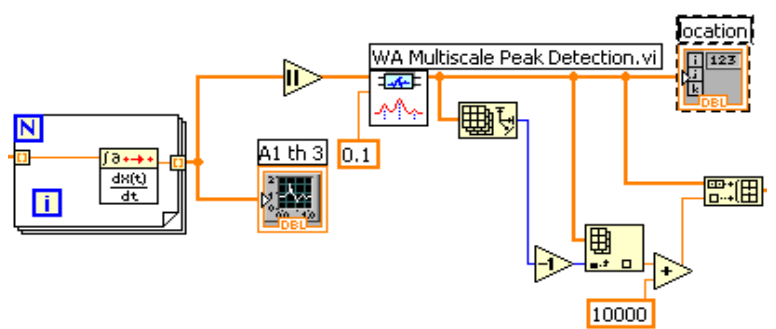

Fig. 6. Localization of HS potions. 
Next is the grouping of HS located nearby points. Fig. 7 shows the block diagram for this purpose. The locations are analyzed and find the portion of original LS to be replaced by the predicted series.

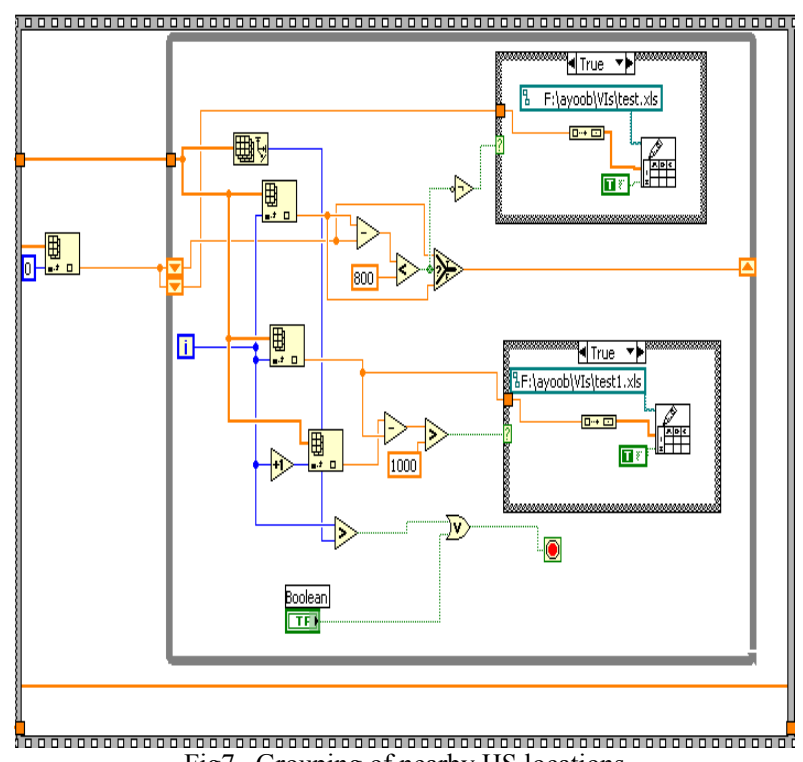

Fig7. Grouping of nearby HS locations.

\section{E. Modeling and Prediction}

The next step was to estimate the removed data by linear prediction, using ARMA models. Here modeling of the LS input and then by using this model, we can predict the values of HS removed portions. TSA ARMA Modeling estimates the autoregressive-moving average (ARMA) model of an input univariate or multivariate (vector) time series according to the method we specify. We can use this polymorphic VI to estimate the ARMA model of waveform, array, vector waveform, and vector array signals. The data type wire to the Xt input determines the polymorphic instance to use.

TSA ARMA Prediction predicts the values of an input univariate or multivariate (vector) time series based on the autoregressive-moving average (ARMA) model. We can use this polymorphic VI to perform ARMA prediction on waveform, array, vector waveform, and vector array signals. The data type wire to the $\mathbf{X t}$ input determines the polymorphic instance to use.

Fig. 8. is the block diagram for ARMA modeling and prediction.

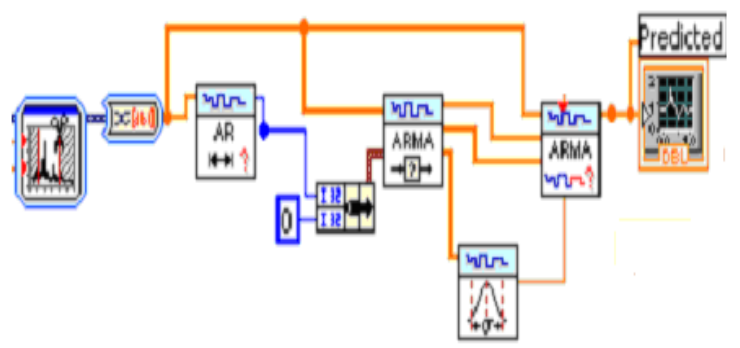

Fig. 8. Block diagram of ARMA modeling and prediction.

\section{Simulation Results}

\section{A. Heart Sound Detection}

The fig. 9 shows the original LS and approximation coefficients of level 1 to level 3.

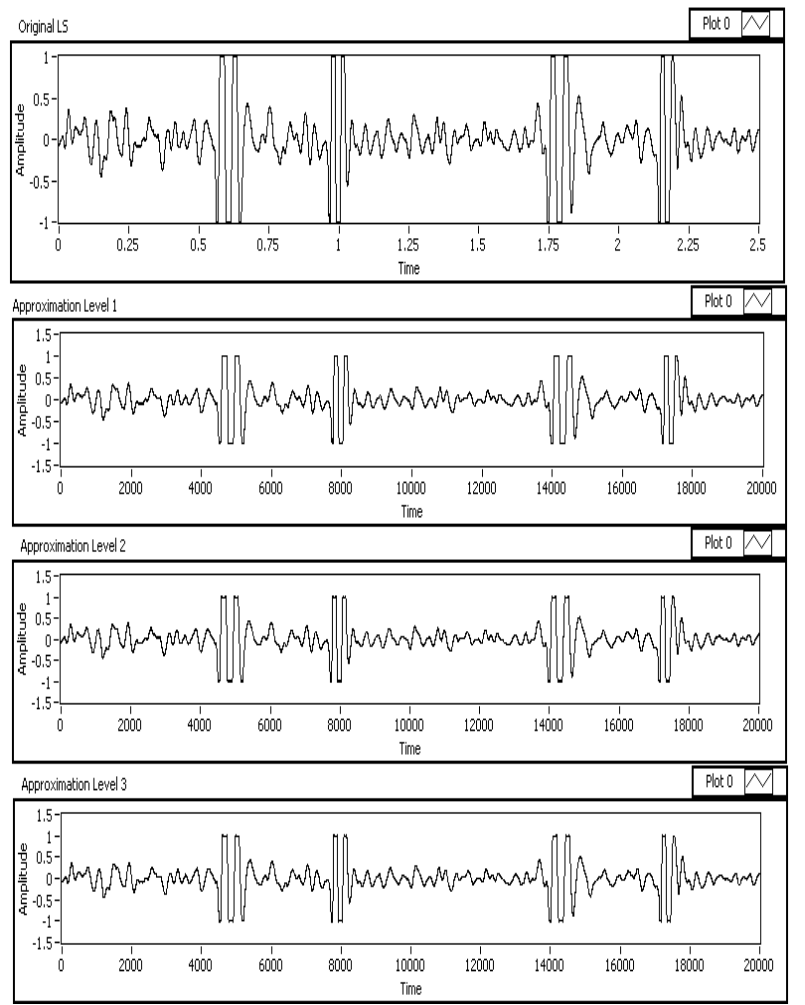

Fig. 9. Original LS and the approximation coefficients at levels 1,2 and 3 respectively.

Fig. 10. is the product of the approximation coefficients of level 1, level 2 and level 3.

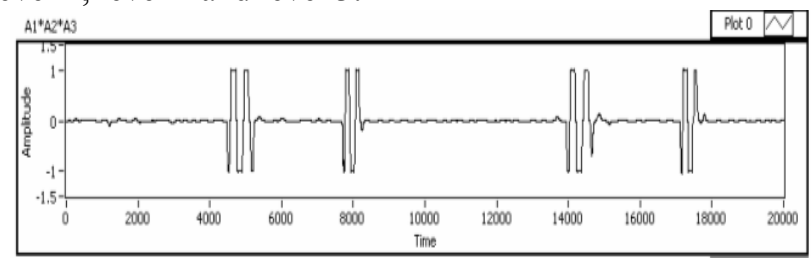

Fig. 10. Multiscale product of approximation coefficients of 1 to 3 levels

\section{B. Heart Sound Cancellation}

HS affected portions of approximation coefficient 1 is removed and it is made as zero which is given in fig. 11 .

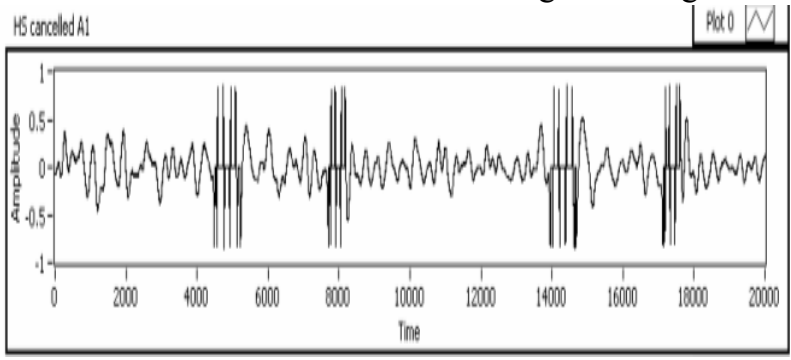

Fig. 11. HS cancelled LS

\section{Modeling and Prediction}

Fig. 12. (bottom) shows the reconstructed LS signal of original LS and signal shown in Fig.12. (top), after HS removal. As it can be seen, all HS segments were removed 
successfully and the main lung sound components were left intact.
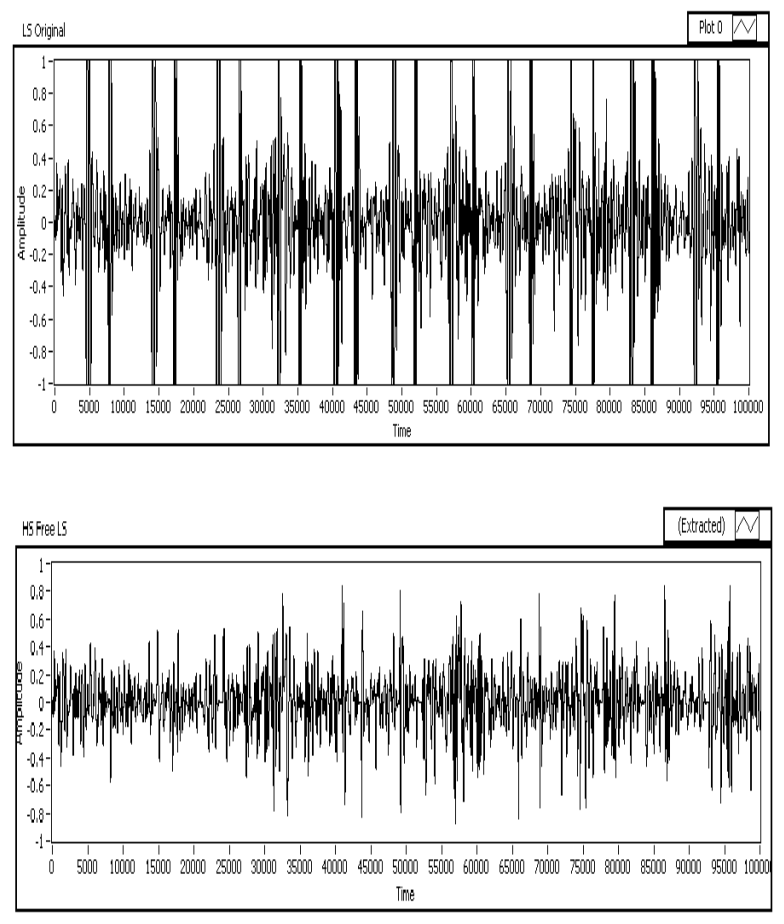

Fig. 12. Original LS( top) and HS separated LS(bottom)

Fig.13. is the expanded views Fig. 12..

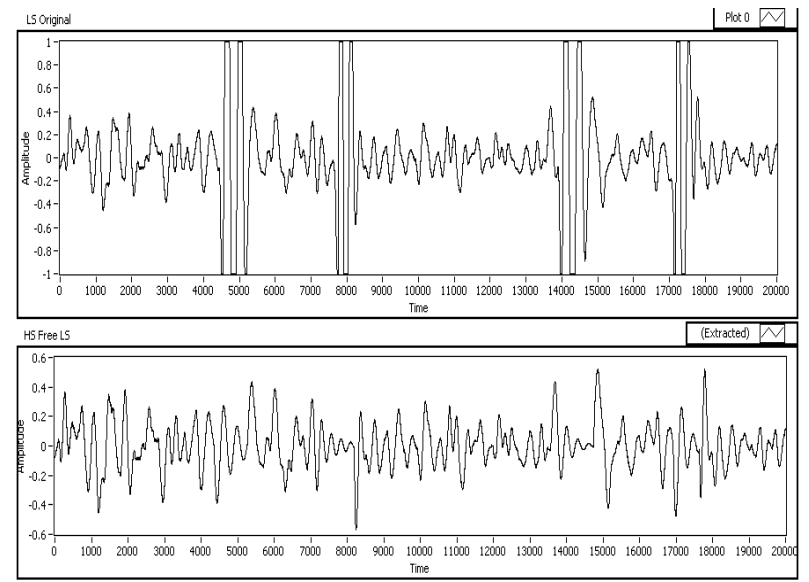

Fig. 13. Original LS (top) and HS separated LS (bottom) for 20000 samples

\section{Validation of Output}

The validation of the result is done by STFT Spectrogram and Power Spectral Density (PSD) analysis of original LS including HS and HS separated LS which are given in Fig. 15 . and Fig. 16. respectively.

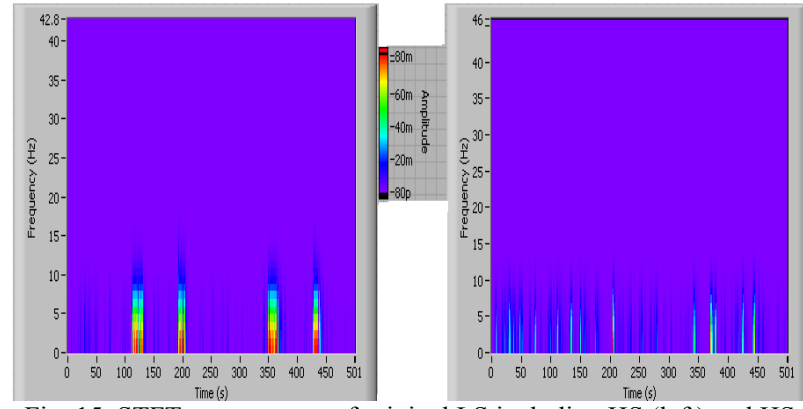

Fig. 15. STFT spectrogram of original LS including HS (left) and HS separated LS (right)

The results of PSD analysis of the original and the reconstructed signals are shown in Fig. 16.

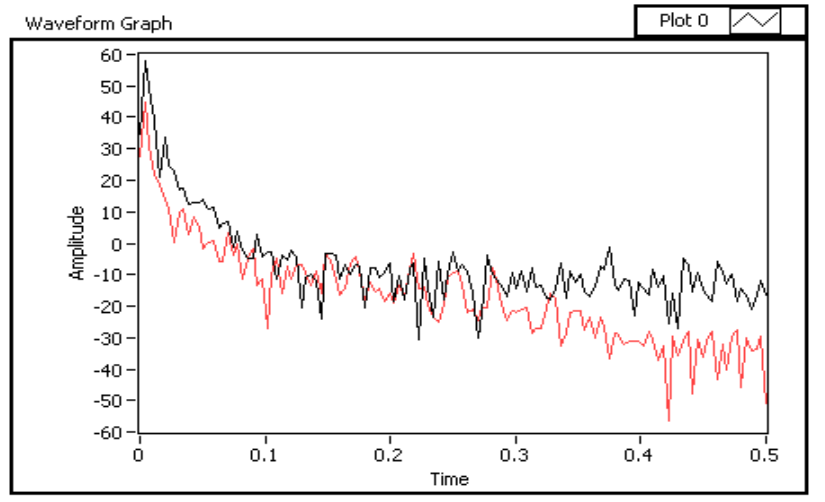

Fig. 16. PSD of original LS including HS (black curve) and HS separated LS (red curve).

\section{CONCLUSION}

The primary objective of respiratory sound research is to bring about improvements to monitoring and diagnosis of respiratory disease, the potential usefulness of any method for filtering heart sounds from lung sounds rests on its ability to perform in a clinical setting. Because the heart and lung sound signals overlap in the time and frequency domains, removing HS interference from respiratory sound recordings is a challenging task. The proposed method for HS removal based on a single recording has shown promising results mainly in terms of lung sound characteristic preservation. This method did not add any noticeable clicks or artifacts in the reconstructed signal. Manual inspection by visual means of the reconstructed signals confirmed that lung sounds were the dominant sounds with no perceptible HS in the background. Furthermore, the proposed technique in this paper is far more efficient than other techniques for HS cancellation in terms of computational load and speed.

This paper presents a novel method for heart sound cancellation from lung sound records using LabVIEW. The method uses the multiscale product of the wavelet coefficients of the original signal to detect HS segments. Once the HS segments are identified, the method removes them from the wavelet coefficients at every level and estimates the created gaps by ARMA model. The results were promising in HS removal from LS without hampering the main components of LS. 


\section{REFERENCES}

[1] V. Gross, A. Dittmar, T. Penzel, F. Schuttler, P. Von Wichert, "The relationship between normal lung sounds, age and gender, “ American Journal of Respiratory and Critical care Medicine 2000; 162: 905-909.

[2] A. Jones, R.D. Jones, K. Kwong, Y. Burns, "Effect of positioning on recorded lung sound intensities in subjects without pulmonary dysfunction," Physical Therapy 1999;79:7,682-690.

[3] F. Dalmay, M.T.Antonini, P.Maequet, R.Menier,'Acoustic properties of the normal chest," European Respiratory Journal 1995; 8:1761-1769.

[4] Z. Moussavi, "Respiratory sound analysis," IEEE in Engineering in Medicine and Biology Magazine, pp15, January/February 2007.

[5] J. Gnitecki, Z. Moussavi, "Seperating heart sounds from lung sounds," IEEE in Engineering in Medicine and Biology Magazine, pp 20-29, January/February 2007.

[6] H. Pasterkamp, S.S. Kraman, and G.R. Wodicka, "Respiratory sounds. advances beyond the stethoscope," Amer. J. Respir. Crit Care Med. vol. 156, no. 3, Pt 1, pp. 974-987, Sept. 1997.

[7] H. Pasterkamp, R. Fenton, A. Tal, and V. Chernick, "Interference of cardiovascular sounds with phonopneumography in children," Am. Rev. Respir. Dis., vol. 131, no. 1, pp. 61-64, Jan. 1985.

[8] I.V. Vovk, V.T. Grinchenko, and V.N. Oleinik, "Modeling the acoustic properties of the chest and measuring breath sounds," Acoustical Physics, vol. 41, no. 5, pp. 667-676, 1995.

[9] I. Hossain and Z. Moussavi, "Relationship between airflow and normal lung sounds," in Proc. 24th Ann. Int. Conf. IEEE Eng. Medicine Biology Soc., EMBC'02, Oct. 2002, pp. 1120-1122.

[10] A.A. Luisada, "The areas of auscultation and the two main heart sounds,"Med.Times, vol. 92, pp. 8-11, Jan. 1964.

[11] P.J. Arnott, G.W. Pfeiffer, and M.E. Tavel, "Spectral analysis of heart sounds: Relationships between some physical characteristics and frequency spectra of first and second heart sounds in normals and hypertensives," J Biomed. Eng., vol. 6, no. 2, pp. 121-128, Apr. 1984.

[12] M. T. Pourazad , Z. Moussavi , G. Thomas,"Heart sound cancellation from lung sound recordings using time-frequency filtering," International Federation for Medical and Biological Engineering 2006 , Med Biol Eng Comput (2006) 44: 216-225.

[13] S. Haykin, Adaptive Filter Theory, 4th ed. Upper Saddle River, NJ: Prentice-Hall, 2002.

[14] Z. Moussavi, D. Flores, and G. Thomas, "Heart sound cancellation based on multiscale products and linear prediction," in Proc. 26th Ann. Int. Conf. IEEE Eng. Medicine Biology Soc., EMBC’04, Sept. 2004 pp. 3840-3843.

[15] I. Hossain and Z. Moussavi, "An overview of heart-noise reduction of lung sound using wavelet transform based filter," in Proc. 25th Ann. Int Conf. IEEE Eng. Medicine Biology Soc., EMBC'03, Sept. 2003, pp. $458-461$.

[16] J. Gnitecki, I. Hossain, Z. Moussavi, and H. Pasterkamp, "Qualitative and quantitative evaluation of heart sound reduction from lung sound recordings," IEEE Trans. Biomed. Eng., vol. 52, no. 10, pp. 1788-1792,Oct. 2005.

[17] S. Mallat and S. Zhong, "Characterization of signals from multiscale edges," IEEE Trans. Pattern Anal. Mach. Intell., vol. 14, no. 7, pp. 710-732, Jul. 1992.

[18] Daniel Flores-Tapia, Zahra M. K. Moussavi, , Gabriel Thomas," Heart sound cancellation based on multiscale products and linear prediction," IEEE Trans. Biomed. Eng., vol. 54, no. 2, pp.234-243,FEBRUARY 2007

[19] http://www.ni.com

[20] http://www.rale.ca

Ayoob Khan T Easa obtained his Bachelors Degree B.Tech (Electronics and Communication Engineering) from Mahatma Gandhi University, Kottayam,India in 1995 and Masters Degree ME (Applied Electronics) from PSG College of Technology, Coimbatore, India in 2008. He is currently with College of Engineering, Kottarakkara, Kerala , India as Lecturer (Selection Grade) in Electronics Engineering Department. He had totally 13 years of teaching experience. He is a member of ISTE. His areas of interests are VLSI Design and Virtual Instrumentation.
Vijayakumar Ponnusamy obtained his Masters Degree ME (Applied Electronics) from PSG College Technology in 2002. He is currently with Karpagam College of Engineering, Coimbatore , India as Professor and Head of the Electrical and Electronics Engineering Department . He had totally around 11 years of teaching experience. He was working in industry for about 7 years between 1992 and 1999. He has published around 10 National and International Journal papers besides 20 papers in Conference proceedings. His areas of interests are Low Power VLSI and Virtual Instrumentation.. 